PROCEEDINGS OF THE

AMERICAN MATHEMATICAL SOCIETY

Volume 133, Number 9, Pages 2795-2803

S 0002-9939(05)07831-7

Article electronically published on March 22, 2005

\title{
SEIBERG-WITTEN INVARIANTS AND BRANCHED COVERS ALONG TORI
}

\author{
B. DOUG PARK
}

(Communicated by Ronald A. Fintushel)

\begin{abstract}
We compute the Seiberg-Witten invariants of double covers of smooth four-manifolds branched along tori of self-intersection zero.
\end{abstract}

\section{INTRODUCTION}

This short paper is a modest generalization of [RW]. In that paper Ruan and Wang computed $(\bmod 2)$ the Seiberg-Witten invariants of double covers of 4manifolds branched along a connected orientable Riemann surface of genus greater than one. In what follows, we shall present a similar $(\bmod 2)$ computation when the branched locus is a torus. Our shared philosophy is to express the Seiberg-Witten invariants of the closed 4-manifolds in terms of the relative invariants of the complements of the branch locus. The computation depends essentially on the gluing formulae for the Seiberg-Witten invariant found in [P2] or [T]. In the last section, we answer in the affirmative a conjecture made by Ruan and Wang in [RW. More examples of our formulae will appear in $\mathrm{P} 3$.

\section{Preliminary Setup AND Definitions}

We shall try to be faithful to the notation in [RW] and [P2]. Let $\widetilde{X}, X$ be closed smooth 4-manifolds and $\Sigma \subset X$ be a smoothly embedded connected orientable surface. We say that a smooth map $p: \widetilde{X} \rightarrow X$ is a cyclic $m$-fold cover branched along $\Sigma$ when the following holds: if $\widetilde{\Sigma}=p^{-1}(\Sigma)$, then the restriction $p: \widetilde{X} \backslash \widetilde{\Sigma} \rightarrow$ $X \backslash \Sigma$ is an unbranched $m$-fold cover, and $p$ has the form $z \mapsto z^{m}$ locally on the normal complex planes of $\widetilde{\Sigma}$ and $\Sigma$.

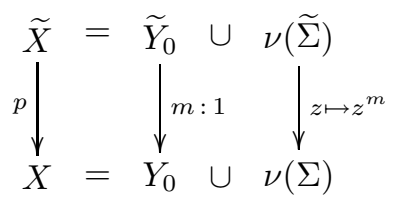

Let $\nu(\Sigma)$ denote a tubular neighborhood of $\Sigma$ and let $Y_{0}:=X \backslash \nu(\Sigma), \widetilde{Y}_{0}:=$ $p^{-1}\left(Y_{0}\right)$. Again, $p$ restricts to an unbranched cover $p: \widetilde{Y}_{0} \rightarrow Y_{0}$. When $m=2$, let $\sigma: \widetilde{Y}_{0} \rightarrow \widetilde{Y}_{0}$ be the covering involution. We shall reserve the $\sim$ symbol for indicating some pull-back object by $p$. As in $[\overline{R W}]$ we will always assume that $[\Sigma]^{2} \geq 0$.

Received by the editors February 5, 2004 and, in revised form, May 3, 2004.

2000 Mathematics Subject Classification. Primary 57M12, 57R57. 
Definition 2.1. (i) Given any complex line bundle $L \rightarrow X$, the virtual dimension with respect to $L$ is defined to be

$$
d_{L}:=\frac{1}{4}\left[c_{1}(L)^{2}-\left(2 e_{X}+3 s_{X}\right)\right]
$$

where $e_{X}$ and $s_{X}$ are respectively the Euler characteristic and the signature of $X$.

(ii) The adjunction term of $\Sigma$ with respect to $L$ is defined to be

$$
J_{L}(\Sigma):=\left|c_{1}(L) \cdot P D[\Sigma]\right|+[\Sigma] \cdot[\Sigma]+e_{\Sigma}
$$

where $P D: H_{2}(X ; \mathbb{Z}) \rightarrow H^{2}(X ; \mathbb{Z})$ is the Poincaré duality isomorphism.

By changing $[\Sigma]$ to $-[\Sigma]$ if necessary, we can always assume that $c_{1}(L) \cdot P D[\Sigma] \leq$ 0 . With that understood, we have the following

Lemma 2.2. (i) $[\widetilde{\Sigma}]^{2}=\frac{1}{m}[\Sigma]^{2}$.

(ii) $e_{\widetilde{X}}=m e_{X}-(m-1) e_{\Sigma}$, and $s_{\widetilde{X}}=m s_{X}-\frac{m^{2}-1}{3}[\widetilde{\Sigma}]^{2}$.

(iii) For any complex line bundle $L \rightarrow X$, we have $c_{1}\left(p^{*} L\right)^{2}=m c_{1}(L)^{2}$.

(iv) Let $\widehat{L}$ be a line bundle such that $c_{1}(\widehat{L})=c_{1}\left(p^{*} L\right)-(m-1) P D[\widetilde{\Sigma}]$. Then $J_{\widehat{L}}(\widetilde{\Sigma})=J_{L}(\Sigma)$.

(v) $d_{\widehat{L}}=m d_{L}+\frac{1}{2}(m-1) J_{L}(\Sigma)$.

Proof. We refer to the excellent book [GS for some of the proofs and [RW] for the special case when $m=2$. The signature formula in part (ii) can be found in $[\mathrm{H}]$. The rest is an easy exercise, which we omit.

From now on we will restrict our attention to the case when the genus of $\Sigma$ is one and $[\Sigma]^{2}=0$. We now briefly recall the setup in [P2]. Suppose we are given a compact 4-manifold $Y_{0}$ with boundary $\partial Y_{0} \cong T^{3}$ and a fixed factorization $\partial Y_{0}=$ $\Sigma \times S^{1}$, where $\Sigma=T^{2}$. Let $\gamma=\left[\{\mathrm{pt}\} \times S^{1}\right] \in H_{1}\left(\partial Y_{0}\right)$, and let $\gamma^{*} \in H^{1}\left(\partial Y_{0}\right)$ be the Poincaré dual of $[\Sigma] \in H_{2}\left(\partial Y_{0}\right)$. Let $X$ be the closed 4-manifold $Y_{0} \cup_{\partial Y_{0}}\left(\Sigma \times D^{2}\right)$, where the boundaries are identified using the above factorization.

Definition 2.3. Let $\gamma$ denote the homology class of the meridian of $\Sigma$ in $\partial Y_{0}$ as above, and let $i: \partial Y_{0} \hookrightarrow Y_{0}$ be the inclusion map. We shall say that the pair $\left(Y_{0}, \Sigma\right)$ is admissible if the following three conditions are satisfied:

(i) $n \gamma \in \operatorname{Ker}\left[i_{*}: H_{1}\left(\partial Y_{0} ; \mathbb{Z}\right) \rightarrow H_{1}\left(Y_{0} ; \mathbb{Z}\right)\right]$ for some positive integer $n$.

(ii) Coker $\left[i^{*}: H^{1}\left(Y_{0} ; \mathbb{Z}\right) \rightarrow H^{1}\left(\partial Y_{0} ; \mathbb{Z}\right)\right]$ is torsion-free.

(iii) The intersection form of $Y_{0}$ is not negative definite.

For an admissible pair $\left(Y_{0}, \Sigma\right)$, we define

$$
\begin{aligned}
& n_{\gamma}:=\min \left\{n>0 \mid n \gamma \in \operatorname{Ker}\left[i_{*}: H_{1}\left(\partial Y_{0} ; \mathbb{Z}\right) \rightarrow H_{1}\left(Y_{0} ; \mathbb{Z}\right)\right]\right\}, \\
& n_{\Sigma}:=\max \left\{n>0 \mid[\Sigma]=n \alpha \text { for some } \alpha \in H_{2}(X ; \mathbb{Z})\right\} .
\end{aligned}
$$

We shall say that the pair $\left(Y_{0}, \Sigma\right)$ is strongly admissible if it is admissible and $n_{\gamma}=n_{\Sigma}$.

Note that condition (i) above implies the existence of an embedded surface $\Gamma_{0} \subset$ $Y_{0}$ such that $\left[\partial \Gamma_{0}\right]=n_{\gamma} \gamma$. Let $\Gamma$ denote the closed connected surface

$$
\Gamma_{0} \cup\left(\coprod_{i=1}^{n_{\gamma}}\left\{\mathrm{pt}_{i}\right\} \times D^{2}\right) \subset X .
$$

It follows that $[\Gamma] \in H_{2}(X ; \mathbb{Z})$ is primitive and $[\Gamma] \cdot[\Sigma]=n_{\gamma} . \Gamma$ is not unique, but we may choose $\Gamma$ so that its genus is minimal among all such surfaces. 
Note that for an $m$-fold branched cover, $m$ divides $n_{\Sigma}$. Let $\Theta$ denote a smoothly embedded surface in $X$ such that $[\Sigma]=n_{\Sigma}[\Theta]$. Let $N$ denote the regular neighborhood of the union $\Theta \cup \Gamma$ inside $X$. If $\left(Y_{0}, \Sigma\right)$ is strongly admissible, then $[\Gamma] \cdot[\Theta]=1$, and hence there is an orthogonal decomposition

$$
H_{2}(X ; \mathbb{Z})=\langle[\Theta],[\Gamma]\rangle \oplus H_{2}(X \backslash N ; \mathbb{Z})
$$

and the corresponding splitting of the intersection form:

$$
Q_{X}=\left[\begin{array}{ll}
0 & 1 \\
1 & c
\end{array}\right] \oplus Q_{X \backslash N}
$$

where $c=[\Gamma] \cdot[\Gamma]$.

If $\left(Y_{0}, \Sigma\right)$ is admissible, then we can define a relative Seiberg-Witten invariant of $Y_{0}$ as follows. Let $\mathcal{S}\left(Y_{0}\right)$ denote the set of isomorphism classes of $\mathrm{Spin}^{c}$ structures on $Y_{0}$ that restricts to the trivial (canonical) $\operatorname{Spin}^{c}$ structure on the boundary $\partial Y_{0}$. Let $\mathcal{H}_{Y_{0}}$ denote the cokernel of the homomorphism $i^{*}: H^{1}\left(Y_{0} ; \mathbb{Z}\right) \rightarrow H^{1}\left(\partial Y_{0} ; \mathbb{Z}\right)$. In $[\mathrm{P} 2]$, we defined a function

$$
S W_{Y_{0}}: \mathcal{S}\left(Y_{0}\right) \times \mathcal{H}_{Y_{0}} \longrightarrow \mathbb{Z},
$$

which algebraically counts the number of solutions to the twice-perturbed SeibergWitten equations for a $\operatorname{Spin}^{c}$ structure $\mathcal{L} \in \mathcal{S}\left(Y_{0}\right)$ on the cylindrical end 4-manifold, $Y:=Y_{0} \cup_{\partial Y_{0}}\left(\partial Y_{0} \times[0, \infty)\right):$

$$
\left\{\begin{array}{l}
\partial_{A} \phi=0, \\
\rho\left(F_{A}+\eta\right)=q(\phi), \\
\eta=f \cdot\left(i h_{(A, \phi)}^{*}(\omega)-i r \pi_{1}^{*} \pi^{*} \mu_{\Sigma}\right) .
\end{array}\right.
$$

We refer the reader to [P2] for the exact definition of the terms in (2.3). The cylindrical end moduli space $\mathcal{M}_{Y}^{r}(\mathcal{L}, g, \omega)$ is defined by dividing the space of finite energy solutions to $(2.3)$ by the action of the $L_{5, \text { loc }}^{2}$ gauge group $\mathcal{G}(Y)$. Note that every solution to 2.3 is irreducible, i.e. $\phi \not \equiv 0$. Recall that there exists a continuous map

$$
\partial_{\infty}: \mathcal{M}_{Y}^{r}(\mathcal{L}, g, \omega) \longrightarrow \mathcal{H}_{Y_{0}}
$$

If $\mathcal{M}_{Y}^{r}(\mathcal{L}, g, \omega)$ is 0 -dimensional, then we define

$$
S W_{Y_{0}}(\mathcal{L}, x):=\#\left[\mathcal{M}_{Y}^{r}(\mathcal{L}, g, \omega) \cap \partial_{\infty}^{-1}(x)\right] .
$$

Definition 2.4. (i) Let $\mathcal{K}_{Y_{0}}$ be the set of isomorphism classes of complex line bundles on $Y_{0}$ that pull back to a trivial bundle on $p^{-1}\left(Y_{0}\right)$. Let $\mathcal{K}_{Y_{0}}^{*}=\mathcal{K}_{Y_{0}} \backslash\{\underline{\mathbb{C}}\}$ be the subset of non-trivial line bundles.

(ii) Suppose $\left(Y_{0}, \Sigma\right)$ is strongly admissible. For any $\operatorname{Spin}^{c}$ structure $\xi$ on $X$ with $\operatorname{det} \xi=L$ and $J_{L}(\Sigma)=-c_{1}(L) \cdot P D[\Sigma]=0$, we let

$$
m_{L}:=c_{1}(L) \cdot P D[\Gamma]
$$

where $\Gamma \subset X$ is defined as in (2.1). We also define

$$
k_{\xi}(X, \Sigma):=\sum_{\kappa \in \mathcal{K}_{Y_{0}}^{*}} \sum_{n=1}^{\infty} S W_{Y_{0}}\left(\left.\xi\right|_{Y_{0}} \otimes \kappa,\left(\llbracket m_{L} / n_{\gamma} \rrbracket-n\right) \gamma^{*}\right),
$$

where $\llbracket m_{L} / n_{\gamma} \rrbracket$ denotes the greatest integer less than or equal to $m_{L} / n_{\gamma}$, and $\gamma^{*}$ is the non-zero element of $\mathcal{H}_{Y_{0}}$ coming from $P D[\Sigma] \in H^{1}\left(\partial Y_{0}\right)$. 
Note that $\mathcal{K}_{Y_{0}}$ is isomorphic to the kernel of the homomorphism $p^{*}: H^{2}\left(Y_{0} ; \mathbb{Z}\right) \rightarrow$ $H^{2}\left(\widetilde{Y}_{0} ; \mathbb{Z}\right)$. Also recall that $c_{1}(\kappa)$ is $m$-torsion for every line bundle $\kappa \in \mathcal{K}_{Y_{0}}$. (See the proof of Theorem 3.8 in [RW] for the case of double covers.) For any complex line bundle $L \rightarrow X$, let us write $c_{1}(L)=a P D[\Theta]+b P D[\Gamma]+\beta$, with $\beta \in H^{2}(X \backslash N ; \mathbb{Z})$ according to the decomposition (2.2). Then $c_{1}(L) \cdot P D[\Sigma]=0$ implies that $b=0$ and $a=m_{L}$. When $b_{2}^{+}(X)>1$, one can show that the sum defining $k_{\xi}(X, \Sigma)$ is finite as in [P1].

\section{MAIN FORMULA}

We now restrict ourselves to the case when $m=2$. The following is a direct generalization of Theorem 6.8 in [RW].

Theorem 3.1. Let $p: \widetilde{X} \rightarrow X$ be a double cover branched along a homologically non-trivial torus $\Sigma \subset X$. Let $Y_{0}=X \backslash \nu(\Sigma)$ be the complement of the tubular neighborhood of $\Sigma$, and let $\widetilde{Y}_{0}=p^{-1}\left(Y_{0}\right), \widetilde{\Sigma}=p^{-1}(\Sigma)$. Assume that $b_{2}^{+}(X), b_{2}^{+}(\widetilde{X})>1$ and $[\Sigma]^{2}=0$. Suppose that $\xi$ is a $\operatorname{Spin}^{c}$ structure on $X$ whose determinant line bundle $L$ satisfies $c_{1}(L) \cdot P D[\Sigma]=0$ and $d_{L}=0$. Let $\mathcal{L}$ denote the restriction $\left.\xi\right|_{Y_{0}}$. If both $\left(\widetilde{Y}_{0}, \widetilde{\Sigma}\right)$ and $\left(Y_{0}, \Sigma\right)$ are admissible, then we have

$$
S W_{\widetilde{Y}_{0}}\left(p^{*}(\mathcal{L}), p^{*}(x)\right) \equiv S W_{Y_{0}}(\mathcal{L}, x)+\sum_{\kappa \in \mathcal{K}_{Y_{0}}^{*}} S W_{Y_{0}}(\mathcal{L} \otimes \kappa, x) \quad \bmod 2 .
$$

Moreover let $\widehat{\xi}$ be a $\operatorname{Spin}^{c}$ structure on $\widetilde{X}$ whose determinant bundle is $\widehat{L}=p^{*} L \otimes$ $P D[\widetilde{\Sigma}]^{-1}$ and whose restriction to $\widetilde{Y}_{0}$ is $p^{*}(\mathcal{L})$. Also define

$$
\Lambda_{L}(\Sigma):=\frac{m_{L}}{n_{\Sigma}}-\llbracket \frac{m_{L}}{n_{\Sigma}} \rrbracket .
$$

Suppose both $\left(\widetilde{Y}_{0}, \widetilde{\Sigma}\right)$ and $\left(Y_{0}, \Sigma\right)$ are strongly admissible. If $\Lambda_{L}(\Sigma) \neq \frac{1}{2}$, then we have

$$
S W_{\widehat{X}}(\widehat{\xi}) \equiv S W_{X}(\xi)+k_{\xi}(X, \Sigma) \bmod 2
$$

If $\Lambda_{L}(\Sigma)=\frac{1}{2}$, then we have

$$
S W_{X}(\xi)+k_{\xi}(X, \Sigma) \equiv 0 \bmod 2 .
$$

Proof. Let $\widetilde{Y}:=\widetilde{Y}_{0} \cup_{\partial \widetilde{Y}_{0}}\left(\partial \widetilde{Y}_{0} \times[0, \infty)\right)$. Consider the following system of equations for the $\operatorname{Spin}^{c}$ structure $p^{*}(\mathcal{L})$ on $\widetilde{Y}$ :

$$
\left\{\begin{array}{l}
\widetilde{\partial}_{A} \phi=0, \\
\rho\left(F_{A}+\widetilde{\eta}\right)=q(\phi), \\
\widetilde{\eta}=(f \circ p) \cdot\left(i h_{(A, \phi)}^{*}\left(p^{*} \omega\right)-i r p^{*} \pi_{1}^{*} \pi^{*} \mu_{\Sigma}\right) .
\end{array}\right.
$$

Here, $\widetilde{\partial}_{A}$ is a twisted Dirac operator constructed from the Levi-Civita connection of the pull-back metric $p^{*} g$ on $\tilde{Y}$. ( $g$ is a generic cylindrical end metric on $Y$ used to define (2.3).) Let $\widetilde{\mathcal{M}}_{\widetilde{Y}}^{r}\left(p^{*} \mathcal{L}, p^{*} g, p^{*} \omega\right)$ denote the solution space of (3.2) divided by the action of a suitable gauge group $\mathcal{G}(\widetilde{Y})$. We can choose $\omega$ such that both $\omega$ and $p^{*} \omega$ are supported away from the critical values of the Chern-Simons-Dirac functional of [P2]. Hence we can prove the compactness the same way and obtain a continuous map

$$
\partial_{\infty}: \widetilde{\mathcal{M}}_{\widetilde{Y}}^{r}\left(p^{*} \mathcal{L}, p^{*} g, p^{*} \omega\right) \longrightarrow \mathcal{H}_{\widetilde{Y}_{0}}
$$


Note that $d_{L}=0$ implies that the virtual dimension of $\mathcal{M}_{Y}^{r}(\mathcal{L}, g, \omega)$ is zero. Since we have also assumed that $J_{L}(\Sigma)=0$, it follows that $d_{\widehat{L}}=0$, and hence the virtual dimension of $\widetilde{\mathcal{M}}_{\widetilde{Y}}^{r}\left(p^{*} \mathcal{L}, p^{*} g, p^{*} \omega\right)$ is also zero. We introduce the notation

$$
\begin{aligned}
\widetilde{\mathcal{N}}_{\widetilde{Y}_{0}}\left(p^{*} \mathcal{L}, p^{*} x ; r, p^{*} g, p^{*} \omega\right) & :=\widetilde{\mathcal{M}}_{\bar{Y}}^{r}\left(p^{*} \mathcal{L}, p^{*} g, p^{*} \omega\right) \cap \partial_{\infty}^{-1}\left(p^{*} x\right), \\
\mathcal{N}_{Y_{0}}(\mathcal{L}, x ; r, g, \omega) & :=\mathcal{M}_{Y}^{r}(\mathcal{L}, g, \omega) \cap \partial_{\infty}^{-1}(x) .
\end{aligned}
$$

Note that there is a well-defined homomorphism $p^{*}: \mathcal{H}_{Y_{0}} \rightarrow \mathcal{H}_{\widetilde{Y}_{0}}$.

Next let $P_{S O(4)}$ denote the frame bundle of $\widetilde{Y}_{0}$, and let $\widetilde{P}$ be the principal $\operatorname{Spin}^{c}(4)$ bundle corresponding to $p^{*} \mathcal{L}$. As in $[\mathrm{RW}]$ we choose a lifting $\tau$ of the covering involution $\sigma$ :

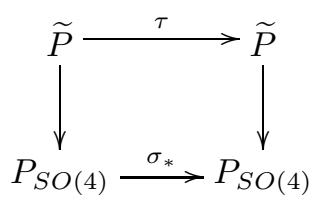

Let $\tau^{*}$ denote the induced action on the Seiberg-Witten configuration space $\mathcal{B}\left(p^{*} \mathcal{L}\right)$. Given any subset $S \subset \mathcal{B}\left(p^{*} \mathcal{L}\right)$, we let $S^{\tau} \subset S$ be the fixed point set of $\tau^{*}: \mathcal{B}\left(p^{*} \mathcal{L}\right) \rightarrow$ $\mathcal{B}\left(p^{*} \mathcal{L}\right)$. Since every solution to (3.2) is irreducible, Theorem 3.8 of [RW] gives us a homeomorphism

$$
\begin{aligned}
& \widetilde{\mathcal{N}}_{\widetilde{Y}_{0}}\left(p^{*} \mathcal{L}, p^{*} x ; r, p^{*} g, p^{*} \omega\right)^{\tau} \\
& \quad \approx \mathcal{N}_{Y_{0}}(\mathcal{L}, x ; r, g, \omega) \coprod\left(\coprod_{\kappa \in \mathcal{K}_{Y_{0}}^{*}} \mathcal{N}_{Y_{0}}(\mathcal{L} \otimes \kappa, x ; r, g, \omega)\right) .
\end{aligned}
$$

Now let $g^{\prime}$ be a generic cylindrical end metric on $\tilde{Y}$. We need to compare the moduli spaces of (2.3) and (3.2) on $\widetilde{Y}$. Since $p^{*} \pi_{1}^{*} \pi^{*} \mu_{\Sigma}=2 \pi_{1}^{*} \pi^{*} \mu_{\widetilde{\Sigma}}$ and we are free to vary the parameter $r \in \mathbb{R} \backslash\{0\}$ in (2.3) (as long as $|r|$ is very small and the sign of $r$ remains the same), we easily see that the only difference between $\mathcal{M}_{\widetilde{Y}}^{2 r}\left(p^{*} \mathcal{L}, g^{\prime}, p^{*} \omega\right)$ and $\widetilde{\mathcal{M}}_{\widetilde{Y}}^{r}\left(p^{*} \mathcal{L}, p^{*} g, p^{*} \omega\right)$ is in the non-generic choice of the metric $p^{*} g$ in the definition of (3.2). Hence Theorem 2.2 of [RW] implies that

$$
\begin{aligned}
S W_{\widetilde{Y}_{0}}\left(p^{*} \mathcal{L}, p^{*} x\right) & =\#\left[\mathcal{N}_{\widetilde{Y}_{0}}\left(p^{*} \mathcal{L}, p^{*} x ; 2 r, g^{\prime}, p^{*} \omega\right)\right] \\
& \equiv \#\left[\widetilde{\mathcal{N}}_{\widetilde{Y}_{0}}\left(p^{*} \mathcal{L}, p^{*} x ; r, p^{*} g, p^{*} \omega\right)^{\tau}\right] \bmod 2 .
\end{aligned}
$$

This proves our first congruence. To prove the second, recall from [P2] (Corollary 20) that

$$
\overline{S W}_{X}=\overline{S W}_{Y_{0}} \cdot\left([\Sigma]+[\Sigma]^{3}+[\Sigma]^{5}+\cdots\right),
$$

where $\overline{S W}$ denotes the Seiberg-Witten series. Hence it follows from the product formula in [P2] that

$$
S W_{X}(\xi)=\sum_{n=1}^{\infty} S W_{Y_{0}}\left(\mathcal{L},\left(\llbracket m_{L} / n_{\Sigma} \rrbracket-2 n+1\right) \gamma^{*}\right) .
$$

(Note that $P D[\Theta] \in H^{2}\left(Y_{0} ; \mathbb{Z}\right) \cong H_{2}\left(Y_{0}, \partial Y_{0} ; \mathbb{Z}\right)$ is $n_{\Sigma}$-torsion.) $\gamma^{*}$ is indivisible and since the difference (modulo torsion) of any two characteristic elements of $H^{2}\left(Y_{0}, \partial Y_{0} ; \mathbb{Z}\right)$ is divisible by two (cf. [MMS], [P1]), we must have by default

$$
S W_{Y_{0}}\left(\mathcal{L},\left(\llbracket m_{L} / n_{\Sigma} \rrbracket-2 n\right) \gamma^{*}\right)=0
$$


for every integer $n$. Thus we may rewrite (3.4) as

$$
S W_{X}(\xi)=\sum_{n=1}^{\infty} S W_{Y_{0}}\left(\mathcal{L},\left(\llbracket m_{L} / n_{\Sigma} \rrbracket-n\right) \gamma^{*}\right) .
$$

Summing our first congruence over $n$, we conclude that

$$
\begin{aligned}
& S W_{X}(\xi)+k_{\xi}(X, \Sigma) \\
& \quad=\sum_{n=1}^{\infty}\left(S W_{Y_{0}}\left(\mathcal{L},\left(\llbracket \frac{m_{L}}{n_{\Sigma}} \rrbracket-n\right) \gamma^{*}\right)+\sum_{\kappa \in \mathcal{K}_{Y_{0}}^{*}} S W_{Y_{0}}\left(\mathcal{L} \otimes \kappa,\left(\llbracket \frac{m_{L}}{n_{\Sigma}} \rrbracket-n\right) \gamma^{*}\right)\right) \\
& \quad \equiv \sum_{n=1}^{\infty} S W_{\widetilde{Y}_{0}}\left(p^{*} \mathcal{L}, p^{*}\left(\llbracket m_{L} / n_{\Sigma} \rrbracket-n\right) \gamma^{*}\right) \bmod 2 .
\end{aligned}
$$

Let $\delta \in H_{1}\left(\partial \widetilde{Y}_{0}\right)$ denote the homology class of the meridian of $\widetilde{\Sigma}$, and let $\delta^{*}:=$ $P D[\widetilde{\Sigma}] \in H^{1}\left(\partial \widetilde{Y}_{0}\right)$. As in (2.1), let $\Delta \subset \widetilde{X}$ be a minimal genus surface such that $[\widetilde{\Sigma}] \cdot[\Delta]=n_{\delta}=n_{\widetilde{\Sigma}}$. Note that $[\Sigma] \cdot p_{*}[\Delta]=2 n_{\delta}=n_{\gamma}$. Since $p^{*}\left(\gamma^{*}\right)=2 \delta^{*}$, we get

$$
S W_{X}(\xi)+k_{\xi}(X, \Sigma) \equiv \sum_{n=1}^{\infty} S W_{\widetilde{Y}_{0}}\left(p^{*} \mathcal{L}, 2\left(\llbracket m_{L} / n_{\Sigma} \rrbracket-n\right) \delta^{*}\right) \quad \bmod 2 .
$$

As in (3.4), we have

$$
S W_{\widetilde{X}}(\widehat{\xi})=\sum_{n=1}^{\infty} S W_{\widetilde{Y}_{0}}\left(p^{*} \mathcal{L},\left(\llbracket m_{\widehat{L}} / n_{\widetilde{\Sigma}} \rrbracket-2 n+1\right) \delta^{*}\right) .
$$

But now note that

$$
m_{\widehat{L}}=c_{1}(\widehat{L}) \cdot P D[\Delta]=-n_{\delta}+c_{1}\left(p^{*} L\right) \cdot P D[\Delta]=-n_{\delta}+m_{L} .
$$

Since $n_{\Sigma}=2 n_{\widetilde{\Sigma}}($ cf. GS] $)$, we must have

$$
\frac{m_{\widehat{L}}}{n_{\widetilde{\Sigma}}}=\frac{-n_{\delta}+m_{L}}{n_{\Sigma} / 2}=-1+\frac{2 m_{L}}{n_{\Sigma}} .
$$

Hence it follows that

$$
S W_{\widetilde{X}}(\widehat{\xi})=\sum_{n=1}^{\infty} S W_{\widetilde{Y}_{0}}\left(p^{*} \mathcal{L},\left(\llbracket 2 m_{L} / n_{\Sigma} \rrbracket-2 n\right) \delta^{*}\right) .
$$

Let $\bar{\xi}$ be the complex conjugate $\operatorname{Spin}^{c}$ structure on $X$ with $\operatorname{det} \bar{\xi}=-L$. We have $S W_{X}(\bar{\xi})=(-1)^{\left(e_{X}+s_{X}\right) / 4} S W_{X}(\xi)$. Hence by changing $\xi$ to $\bar{\xi}$ if necessary, we may assume that

$$
0 \leq \frac{m_{L}}{n_{\Sigma}}-\llbracket \frac{m_{L}}{n_{\Sigma}} \rrbracket \leq \frac{1}{2}
$$

Now if $m_{L} / n_{\Sigma}-\llbracket m_{L} / n_{\Sigma} \rrbracket \neq 1 / 2$, then we get

$$
\llbracket 2 m_{L} / n_{\Sigma} \rrbracket=2 \llbracket m_{L} / n_{\Sigma} \rrbracket .
$$

Thus the combination of (3.6) and (3.7) proves our second congruence, provided that $m_{L} / n_{\Sigma}-\llbracket m_{L} / n_{\Sigma} \rrbracket \neq 1 / 2$. Finally suppose that

$$
m_{L}=\llbracket \frac{m_{L}}{n_{\Sigma}} \rrbracket n_{\Sigma}+\frac{n_{\Sigma}}{2} .
$$


Then we have $\llbracket 2 m_{L} / n_{\Sigma} \rrbracket=2 \llbracket m_{L} / n_{\Sigma} \rrbracket+1$, and thus

$$
S W_{\widehat{X}}(\widehat{\xi})=\sum_{n=1}^{\infty} S W_{\widetilde{Y}_{0}}\left(p^{*} \mathcal{L},\left(2 \llbracket m_{L} / n_{\Sigma} \rrbracket+1-2 n\right) \delta^{*}\right) .
$$

As in (3.5), it follows that

$$
S W_{\widetilde{Y}_{0}}\left(p^{*} \mathcal{L},\left(2 \llbracket m_{L} / n_{\Sigma} \rrbracket-2 n\right) \delta^{*}\right)=0
$$

for every integer $n$. Hence (3.6) implies that

$$
S W_{X}(\xi)+k_{\xi}(X, \Sigma) \equiv 0 \bmod 2 .
$$

Remark 3.2. (i) If $b_{2}^{+}(X)>1$, then by the adjunction inequality (cf. $\left.\mathrm{OS}\right]$ ), $c_{1}(L)$. $P D[\Sigma] \neq 0$ implies that $S W_{X}(\xi)=0$. Since $c_{1}(\widehat{L}) \cdot P D[\widetilde{\Sigma}]=c_{1}(L) \cdot P D[\Sigma]$, it also implies that if $b_{2}^{+}(\widetilde{X})>1$, then we must have $S W_{\widetilde{X}}(\widehat{\xi})=0$ as well.

(ii) If $c_{1}(L) \cdot P D[\Sigma]=0$ but $d_{L} \neq 0$, then by Lemma 2.2 (v) we have $d_{\widehat{L}} \neq 0$. Hence if $\widetilde{X}$ is of Seiberg-Witten simple-type (e.g. if $\widetilde{X}$ is a symplectic manifold), then $S W_{\widetilde{X}}(\widehat{\xi})=0$.

(iii) Let $\lambda \rightarrow X$ be the complex line bundle with $c_{1}(\lambda)=\frac{1}{2} P D[\Sigma]$ determined by the branched cover $p: \widetilde{X} \rightarrow X$. If $\left(Y_{0}, \Sigma\right)$ is admissible, then $[\Sigma] \in H_{2}(X ; \mathbb{Z})$ is not torsion. Hence Proposition 5.3 of $[\overline{\mathrm{RW}}]$ implies that $\left.\lambda\right|_{Y_{0}} \in \mathcal{K}_{Y_{0}}^{*}$, and if $H_{1}(X ; \mathbb{Z})$ contains no 2-torsion, then $\mathcal{K}_{Y_{0}}^{*}=\left\{\left.\lambda\right|_{Y_{0}}\right\}$.

\section{EXAMPLES}

We start out with the following conjecture made by Ruan and Wang:

Conjecture 4.1 (cf. [RW], p. 502). The difference of the $S W$-invariants,

$$
S W_{\widetilde{X}}(\widehat{\xi})-S W_{X}(\xi) \bmod 2,
$$

is not always zero.

We prove the conjecture (at least when the genus of $\Sigma$ is one) by providing examples with $k_{\xi}(X, \Sigma) \equiv 1 \bmod 2$.

For each integer $n>0$, let $E(n)$ be a simply-connected elliptic surface with no multiple fibers and with geometric genus $p_{g}=n-1$. Let $F$ denote a generic torus fiber of $E(n)$, and let $E(n)_{q}$ be the result of a logarithmic transformation of multiplicity $q>1$ along $F$. Let $F_{q} \subset E(n)_{q}$ denote the multiple fiber. Recall that $[F]=q\left[F_{q}\right]$ in $H_{2}\left(E(n)_{q} ; \mathbb{Z}\right)$. If $q=2 \ell$ is even, then let $\widetilde{E}(n)_{q}$ be the double cover of $E(n)_{q}$ branched along the torus $\Sigma=F$.

We may take $\Gamma$ to be the union of a normal disk of $F_{q}$ and $q$ punctured sections in $E(n) \backslash \nu(F)$, and set $\Theta=F_{q}$. It is not very hard to check that the pairs $\left(\widetilde{E}(n)_{q} \backslash\right.$ $\nu(\widetilde{F}), \widetilde{F})$ and $\left(E(n)_{q} \backslash \nu(F), F\right)$ are strongly admissible. (Note that $\mathcal{H}_{\widetilde{E}(n)_{q} \backslash \nu(\widetilde{F})} \cong$ $\mathcal{H}_{E(n)_{q} \backslash \nu(F)} \cong \mathbb{Z}^{3}$.)

Lemma 4.2. Let $t=[F]$ and $t_{q}=\left[F_{q}\right]$ in $H_{2}\left(E(n)_{q} ; \mathbb{Z}\right)$. Then we have

$$
\begin{gathered}
\overline{S W}_{E(n)_{q}}=\left(t^{-1}-t\right)^{n-2} \cdot\left(t_{q}^{q-1}+t_{q}^{q-3}+\cdots+t_{q}^{-(q-3)}+t_{q}^{-(q-1)}\right), \\
\overline{S W}_{E(n)_{q} \backslash \nu(F)}=\left(t^{-1}-t\right)^{n-1} \cdot\left(t_{q}^{q-1}+t_{q}^{q-3}+\cdots+t_{q}^{-(q-3)}+t_{q}^{-(q-1)}\right) .
\end{gathered}
$$

Proof. $\overline{S W}_{E(n)_{q}}$ was computed in [FS]. Apply Corollary 20 of [P2] to it. (See formula (3.3. above.) 
Theorem 4.3. Let $\xi_{j}$ denote the $\operatorname{Spin}^{c}$ structure on $E(2)_{2 \ell}$ with $c_{1}\left(\operatorname{det} \xi_{j}\right)=$ $P D\left(j\left[F_{2 \ell}\right]\right)$. If $j$ is odd and satisfies $1 \leq j \leq 2 \ell-1$, then

$$
k_{\xi_{j}}\left(E(2)_{2 \ell}, F\right)=1 \text {. }
$$

Moreover, if $j \neq \ell$, then

$$
S W_{\widetilde{E}(2)_{2 \ell}}\left(\widehat{\xi}_{j}\right)-S W_{E(2)_{2 \ell}}\left(\xi_{j}\right) \equiv 1 \bmod 2 .
$$

Therefore Conjecture 4.1 is true.

Proof. As before let $Y_{0}=E(2)_{2 \ell} \backslash \nu(F)$ and let $L_{j}=\operatorname{det} \xi_{j}$. Let $t=[F]$ and $t_{q}=\left[F_{2 \ell}\right]$ with $q=2 \ell$. Note that $n_{F}=2 \ell$ and $m_{L_{j}}=j$. Thus $\llbracket m_{L_{j}} / n_{F} \rrbracket=0$. Since $E(n)_{q}$ is simply-connected, $\mathcal{K}_{Y_{0}}^{*}=\left\{\left.\lambda\right|_{Y_{0}}\right\}$, where $c_{1}(\lambda)=\ell P D\left(t_{q}\right)$. Thus if $1 \leq j \leq 2 \ell-1$, we have

$$
k_{\xi_{j}}\left(E(2)_{2 \ell}, F\right)=\sum_{n=1}^{\infty} S W_{Y_{0}}\left(\left.\left.\xi_{j}\right|_{Y_{0}} \otimes \lambda\right|_{Y_{0}},(-n) \gamma^{*}\right) .
$$

Now recall from [P2] that

$$
S W_{Y_{0}}\left(\left.\left.\xi_{j}\right|_{Y_{0}} \otimes \lambda\right|_{Y_{0}}, m \gamma^{*}\right)=S W_{Y_{0}}\left(\left.\xi_{j}\right|_{Y_{0}},(m-1) \gamma^{*}\right)
$$

for every integer $m$. Hence we conclude that

$$
k_{\xi_{j}}\left(E(2)_{2 \ell}, F\right)=\sum_{n=1}^{\infty} S W_{Y_{0}}\left(\left.\xi_{j}\right|_{Y_{0}},(-n-1) \gamma^{*}\right) .
$$

From Lemma 4.2, we have

$$
\overline{S W}_{Y_{0}}=\left(t^{-1}-t\right) \cdot\left(t_{q}^{q-1}+t_{q}^{q-3}+\cdots+t_{q}^{-(q-3)}+t_{q}^{-(q-1)}\right) .
$$

Let $a_{n, j}$ denote the coefficient of $t^{n} t_{q}^{j}$ in $\overline{S W}_{Y_{0}}$ with $|j| \leq q-1$. From the definition of a Seiberg-Witten series in [P2], we deduce that if $|j| \leq q-1$, then

$$
a_{n, j}=S W_{Y_{0}}\left(\left.\xi_{j}\right|_{Y_{0}}, n \gamma^{*}\right) \text {. }
$$

It follows that if $j$ is odd and $1 \leq j \leq 2 \ell-1$, then

$$
k_{\xi_{j}}\left(E(2)_{2 \ell}, F\right)=\sum_{n=1}^{\infty} a_{-n-1, j}=a_{-2, j}=a_{-1, j-2 \ell}=1 .
$$

For the last statement note that if $1 \leq j \leq 2 \ell-1$ and $j \neq \ell$, then

$$
\Lambda_{L_{j}}(F)=\frac{m_{L_{j}}}{n_{F}}-\llbracket \frac{m_{L_{j}}}{n_{F}} \rrbracket=\frac{j}{2 \ell}-\llbracket \frac{j}{2 \ell} \rrbracket=\frac{j}{2 \ell} \neq \frac{1}{2} .
$$

Now apply congruence (3.1) of Theorem 3.1.

Remark 4.4. (i) For $n>2$, let $\xi_{i, j}$ be the $\operatorname{Spin}^{c}$ structure on $E(n)_{2 \ell}$ such that $c_{1}\left(\operatorname{det} \xi_{i, j}\right)=P D\left(i t+j t_{q}\right)$ with $|j|<2 \ell$. As in the last proof, we can express $k_{\xi_{i, j}}\left(E(n)_{2 \ell}, F\right)$ as a sum of coefficients of $\overline{S W}_{E(n)_{2 \ell} \backslash \nu(F)}$. In this way we can find a plethora of instances where $k_{\xi_{i, j}}\left(E(n)_{2 \ell}, F\right) \equiv S W_{\widetilde{E}(n)_{2 \ell}}\left(\widehat{\xi}_{i, j}\right)-S W_{E(n)_{2 \ell}}\left(\xi_{i, j}\right) \equiv 1$ $\bmod 2$.

(ii) If $\ell$ is odd and $j=\ell$, then $\Lambda_{L_{j}}(F)=\frac{1}{2}$ and $S W_{E(2)_{2} \ell}\left(\xi_{j}\right)=1$. Thus we have

$$
S W_{E(2)_{2 \ell}}\left(\xi_{j}\right)+k_{\xi_{j}}\left(E(2)_{2 \ell}, F\right)=2 \equiv 0 \bmod 2,
$$

which is the final congruence of Theorem 3.1 .

(iii) Many more examples with $k_{\xi}(X, \Sigma) \equiv 1 \bmod 2$ can be found in [P3]. 


\section{ACKNOWLEDGMENTS}

The author would like to thank Ian Hambleton for introducing him to the field of equivariant gauge theory and providing inspiration. He would also like to thank Tian-Jun Li and Michael McCooey for very helpful discussions.

\section{REFERENCES}

[FS] R. Fintushel and R. J. Stern: Rational blowdowns of smooth 4-manifolds, J. Differential Geom. 46 (1997), 181-235. MR1484044 (98j:57047)

[GS] R. E. Gompf and A. I. Stipsicz: 4-Manifolds and Kirby Calculus, Graduate Studies in Mathematics, No. 20, Amer. Math. Soc., 1999. MR1707327 (2000h:57038)

[H] F. Hirzebruch: The signature of ramified coverings, in Global Analysis (Papers in Honor of K. Kodaira), pp. 253-265, Univ. Tokyo Press, Tokyo, 1969. MR0258060 (41:2707)

[MMS] J. W. Morgan, T. S. Mrowka and Z. Szabó: Product formulas along $T^{3}$ for Seiberg-Witten invariants, Math. Res. Lett. 4 (1997), 915-929. MR.1492130 (99f:57039)

[OS] P. Ozsváth and Z. Szabó: Higher type adjunction inequalities in Seiberg-Witten theory, J. Differential Geom. 55 (2000), 385-440. MR1863729 (2002j:57061)

[P1] B. D. Park: A product formula for the Seiberg-Witten invariant along certain Seifert fibered manifolds, Asian J. Math. 6 (2002), 37-60. MR1902646 (2003b:57047)

[P2] B. D. Park: A gluing formula for the Seiberg-Witten invariant along $T^{3}$, Michigan Math. J. 50 (2002), 593-611. MR1935154 (2003i:57051)

[P3] B. D. Park: Constraints on Alexander polynomials of certain two-component links, Topology Appl. 144 (2004), 161-171. MR2097134

[RW] Y. Ruan and S. Wang: Seiberg-Witten invariants and double covers of 4-manifolds, Comm. Anal. Geom. 8 (2000), 477-515. MR1775135 (2001h:57039)

[T] C. H. Taubes: The Seiberg-Witten invariants and 4-manifolds with essential tori, Geom. Topol. 5 (2001), 441-519. MR1833751 (2002d:57025)

Department of Pure Mathematics, University of Waterloo, Waterloo, Ontario, CANAdA N2L 3G1

E-mail address: bdpark@math.uwaterloo.ca

URL: http://www.math.uwaterloo.ca/ bdpark/ 